\title{
Development of a portable 3D-printed flow-through passive sampling device free of flow pattern effects
}

Fidelis Nitti ${ }^{a, b}$, M. Inês G.S. Almeida a,b, Richard Morrison ${ }^{a}$, Robert W. Cattrall a, Vincent J. Pettigrove ${ }^{\mathrm{c}}$, Rhys A. Coleman ${ }^{\mathrm{d}}$, Spas D. Kolev ${ }^{\mathrm{a}, \mathrm{b},{ }^{*}}$

${ }^{a}$ School of Chemistry, The University of Melbourne, VIC 3010, Australia

${ }^{\mathrm{b}}$ Centre for Aquatic Pollution Identification and Management (CAPIM), School of Chemistry, The University of Melbourne, Victoria 3010, Australia

${ }^{\mathrm{c}}$ Centre for Aquatic Pollution Identification and Management (CAPIM), School of Biosciences, The University of Melbourne, Victoria 3010, Australia

${ }^{\mathrm{d}}$ Melbourne Water Corporation, Docklands, Melbourne, Victoria 3008, Australia

\begin{abstract}
A low cost and portable flow-through passive sampling device (PSD) which is not affected by the flow pattern of the sampled aquatic system was developed. $\mathrm{Zn}^{2+}$ was selected as the target analyte in optimizing and testing the device under laboratory conditions using as the source solution (SS) either $\mathrm{Zn}^{2+}$ solutions or environmental waters, spiked with $\mathrm{Zn}^{2+}$. It was demonstrated that the accumulated amount of $\mathrm{Zn}^{2+}$ in all sampling experiments with the newly developed PSD was independent of the flow pattern of the sampled SS which was in contrast with results obtained with previously developed dip-in and shielded dip-in passive samplers. The flow-through PSD consists of an acrylic-based 3D-printed flow-through compartment, a glass vessel containing the receiving
\end{abstract}

\footnotetext{
*Corresponding author. Tel.: +61 38344 7931; Email: s.kolev@unimelb.edu.au
} 
solution (RS) and a polymer inclusion membrane which acts as a semipermeable barrier separating the RS from a stream of the sampled water (i.e. SS). The PIM was composed of $35 \mathrm{wt} \%$ commercial dinonylnaphthalene sulfonic acid (DNNS), $55 \mathrm{wt} \%$ poly(vinyl chloride) and $10 \mathrm{wt} \%$ 1-tetradecanol. Though in some of the experiments the SS was propelled through the PSD by a conventional peristaltic pump, unsuitable for use in the field, it was demonstrated that the replacement of the peristatic pump with a small battery-driven piezoelectric micro pump was straightforward and did not affect the performance of the PSD. This finding proves the suitability of the newly developed flow-through PSD as a cost effective and portable tool for passive sampling of environmental waters free of flow pattern effects.

Keywords: passive sampling; polymer inclusion membrane; zinc; flow pattern effects 


\section{Introduction}

Passive sampling is rapidly gaining attention as an alternative environmental monitoring technique which facilitates the determination of the time-weighted average (TWA) concentrations of various pollutants in aquatic systems [1-5]. Unlike spot sampling which relies on the collection of discrete water samples and their subsequent laboratory analysis, passive sampling involves the continuous deployment of sampling devices in the water body of interest for a certain period of time. During this period, passive accumulation of a specific chemical species of interest occurs continuously, thus allowing the detection of episodic pollution events over that period that might otherwise be missed by routine spot sampling. Passive sampling can also combine the sampling process with elimination of matrix interferences, analyte pre-concentration and sample preservation, thus allowing the use of less sensitive analytical instruments for the final analytical measurement $[2$, 6]. Moreover, the devices used for passive sampling are usually small, easy to fabricate, inexpensive and easily deployable in aquatic systems by personnel with minimal training. The above mentioned features provide great advantages in sampling of aquatic systems which are not easily accessible [3].

Since their introduction over three decades ago, numerous passive sampling devices (PSDs) have been developed and commercialized. Most passive samplers typically consist of a receiving phase which collects the analyte of interest and a semipermeable barrier which separates the receiving phase from the sampled medium [6]. Currently, the passive sampling of metal ions in aquatic systems is mainly conducted by either diffusive gradient in thin films (DGT) or Chemcatcher passive samplers [7-10]. The processing of these devices, however, requires a complex and timeconsuming pretreatment step involving the back-extraction of the analyte from the solid receiving phase prior to its final determination which increases analytical costs and errors in determining the 
TWA concentrations. A passive sampling approach incorporating a supported liquid membrane (SLM) as the semipermeable barrier and a liquid receiving phase has been tested to overcome this limitation based on the fact that the measuring of the metal ion(s) concentration in the liquid receiving phase can be done directly (i.e., without an elution step) [11-13]. However, the practical applicability of this approach is limited by the fact that an SLM, consisting of a microporous hydrophobic membrane with an organic solution of a suitable extractant retained in its pores by week capillary force, has a relatively short life-time due to the easy leaching of the organic solution into the aquatic system during the deployment period [14].

Polymer inclusion membranes (PIMs) are the most recent type of liquid membranes. They differ from SLMs in that they incorporate the liquid organic extractant within the entangled chains of the base polymer structure $[15,16]$. This is the reason why PIMs have greater stability and longer lifetime in comparison to their SLM counterparts $[17,18]$. Similarly to the SLMs, PIMs also allow the extraction and back-extraction of the target analyte to occur simultaneously on each side of the membrane, thus making them suitable as semipermeable barriers in PSDs. Another major advantage of using PIMs in passive sampling is the versatility they offer in terms of selectivity. The composition of a PIM can be selected to provide selectivity for the target analyte being studied. Moreover, the composition of the receiving solution can be adjusted to provide rapid stripping of the analyte from the PIM and an adequate capacity to accommodate a sufficient amount of analyte thus preventing its saturation during the sampling period. A number of recent research articles have demonstrated the potential of PIMs in passive sampling $[15,16,19]$.

Even though passive sampling offers great advantages, the reliability of the TWA measurements of a certain pollutant in environmental waters is strongly influenced by environmental conditions, particularly the flow pattern at the deployment site. A few studies have concluded that the 
hydrodynamics conditions at the deployment site can influence significantly the passive sampling results as they control the thickness of the water boundary layer at the passive sampler's membrane/aquatic system interface through which the analyte diffuses towards the membrane [1921]. Ideally, a laboratory-based calibration with hydrodynamic conditions comparable to the field conditions should be undertaken. However, this is laborious, time-consuming and unreliable considering that it is practically impossible to mimic the unpredictable flow patterns of aquatic systems under laboratory conditions, which can lead to significant uncertainties in the results obtained by existing PSDs [22]. A number of approaches have been proposed to correct for flow pattern effects in passive sampling. One of the most promising methods is based on the use of performance reference compounds (PRC). PRCs are loaded on the passive samplers prior to their deployment and the effect of the flow pattern is quantified on the basis of the dissipation rate of the PRC [1]. This technique has been successfully applied for several passive samplers and its theory has also been adequately developed, especially for the sampling of non-polar organic compounds [23]. However, the unavailability of suitable PRCs for the passive sampling of polar organic compounds and metal ions has been reported to be its main drawback [24, 25]. An alternative approach for correcting the effect of the flow pattern on the passive sampling results has recently been described and it is based on the use of a passive flow monitor (PFM). This approach involves the co-deployment of the passive samplers and a PFM which consists of calcium sulphate dihydrate (gypsum) and the correction of flow pattern effects on the passive sampling results is calculated on the basis of the mass loss of the PFM [26].

Other approaches aiming at eliminating the flow pattern effects on passive sampling results include the introduction of constant turbulence around the passive samplers or inducing a constant flow of the source solution through the passive samplers during the period of deployment. Llorca et al. 
developed two passive samplers for the sampling of six polycyclic aromatic hydrocarbons and three organochlorine pesticides [27]. One of the samplers was a constantly stirred sorbent while the other one was a continuous flow integrative sampler. The constant turbulence created around the constantly stirred sorbent sampler and the stable flow of the source solution through the second passive sampler resulted in constant mass transfer of the analytes to the corresponding receiving phases, thus making them independent of the surrounding hydrodynamic conditions. The continuous flow integrative sampler was found to be more reliable. Petersen et al. [28] described a mobile continuous flow system utilizing a Chemcatcher sampler for the monitoring of rare earth elements in marine environments. Like the previous two passive samplers, this passive sampler was based on a constant flow of the source solution through the flow cell containing the Chemcatcher sampler, thus maintaining a constant uptake of the analytes irrespective of the flow pattern. However, the above mentioned approaches involve relatively big devices with limited portability.

In the present paper, we describe the development of a small portable flow-through PSD whose performance is unaffected by the flow pattern of the sampled medium (source solution). This newly developed PSD utilizes a PIM as the semipermeable barrier and an acidic solution as the receiving phase, which can be analyzed directly (no elution required). $\mathrm{Zn}^{2+}$ was chosen as the target metal ion for this study as it is frequently encountered in environmental waters and depending on its concentration it can be highly toxic to aquatic organisms and humans [29, 30].

\section{Material and methods}

\subsection{Reagents and solutions}

All chemical reagents used in this research were of analytical reagent grade and were used as 
received without any further purification. Deionized water (Milipore, Synergy 185, France, resistivity $\geq 18.2 \mathrm{M} \Omega \mathrm{cm}$ ) was used to prepare all aqueous solutions if not stated otherwise.

A $500 \mathrm{~mL}$ of primary stock solution containing $1000 \mathrm{mg} \mathrm{L}^{-1} \mathrm{Zn}^{2+}$ was prepared by dissolving the required amount of $\mathrm{ZnCl}_{2}$ (Unilab, Australia), which had been previously dried at $100{ }^{\circ} \mathrm{C}$ for $2 \mathrm{~h}$. A secondary $\mathrm{Zn}^{2+}$ stock solution was prepared by a ten-fold dilution of the primary stock solution in deionized water, yielding a final concentration of $100.0 \mathrm{mg} \mathrm{L}^{-1}$. The source solutions containing $\mathrm{Zn}^{2+}$ used in all the passive sampling experiments were prepared by an appropriate dilution of the secondary stock solution in a $5.00 \mathrm{~L}$ volumetric flask using either deionized water or environmental water.

The receiving solution in all the passive sampling experiments consisted of $1.0 \mathrm{~mol} \mathrm{~L}^{-1} \mathrm{HNO}_{3}$ solution and was prepared by dilution of concentrated $\mathrm{HNO}_{3}$ (70\%, Ajax, Australia). A series of $\mathrm{Zn}^{2+}$ standard solutions for the determination of $\mathrm{Zn}^{2+}$ concentration using atomic absorption spectroscopy (AAS) were prepared prior to each analysis by dilution of high purity standards containing $100.0 \mathrm{mg} \mathrm{L}^{-1} \mathrm{Zn}^{2+}$ (Choice Analytical, Australia) in 5.0\% $\mathrm{HNO}_{3}$ solution.

A series of standard solutions for the inductively coupled plasma optical emission spectrometry (ICP-OES) measurements of interfering cations in spiked environmental water samples were prepared by dilution of the following stock solutions: sodium chloride (Chem-supply, Australia) containing $50.0 \mathrm{~g} \mathrm{~L}^{-1} \mathrm{Na}^{+}$, potassium hydrogen carbonate (Ajax, Australia) containing $10.0 \mathrm{~g} \mathrm{~L}^{-1}$ $\mathrm{K}^{+}$, calcium nitrate tetrahydrate (Chem-supply, Australia) containing $20.0 \mathrm{~g} \mathrm{~L}^{-1} \mathrm{Ca}^{2+}$, magnesium sulphate heptahydrate (Chem-supply, Australia) containing $15.0 \mathrm{~g} \mathrm{~L}^{-1} \mathrm{Mg}^{2+}$, aluminium chloride hexahydrate (Chem-supply, Australia) containing $0.500 \mathrm{~g} \mathrm{~L}^{-1} \mathrm{Al}^{3+}$, and ferrous sulphate heptahydrate (Ajax, Australia) containing $0.500 \mathrm{~g} \mathrm{~L}^{-1} \mathrm{Fe}^{2+}$. 
The following components were used to prepare the PIMs used in this research: PVC (Aldrich) as the base polymer, dinonylnaphthalene sulfonic acid (DNNS, King Industries, $50 \mathrm{wt} \%$ in 2butoxyethanol) as the extractant and 1-tetradecanol (Aldrich) as a modifier. Tetrahydrofuran without a stabilizer (VWR, Australia) was used as the solvent in membrane casting.

Freshwater collected from Cairn Curran Reservoir (Victoria, Australia) was spiked with $\mathrm{Zn}^{2+}$ and used as a source solution in the passive sampling experiments aimed at comparing the performance of piezoelectric pumps with a peristaltic pump. This freshwater sample was characterized in terms of its chemical composition and conductivity (Table 1).

Table 1. Chemical composition and conductivity of freshwater collected from Cairn Curran Reservoir.

\begin{tabular}{lc}
\hline Chemical parameters & Value \\
\hline $\mathrm{pH}$ & $6.61 \pm 0.02$ \\
Conductivity $\left(\mu \mathrm{S} \mathrm{cm}^{-1}\right)$ & $307 \pm 2$ \\
$\mathrm{Na}^{+}$concentration $\left(\mathrm{mg} \mathrm{L}^{-1}\right)$ & $33.5 \pm 0.8$ \\
$\mathrm{~K}^{+}$concentration $\left(\mathrm{mg} \mathrm{L}^{-1}\right)$ & $4.41 \pm 0.09$ \\
$\mathrm{Mg}^{2+}$ concentration $\left(\mathrm{mg} \mathrm{L}^{-1}\right)$ & $13.3 \pm 0.4$ \\
$\mathrm{Ca}^{2+}$ concentration $\left(\mathrm{mg} \mathrm{L}^{-1}\right)$ & $7.69 \pm 0.29$ \\
$\mathrm{Fe}^{3+}$ concentration $\left(\mathrm{mg} \mathrm{L}^{-1}\right)$ & $2.61 \pm 0.25$ \\
$\mathrm{Al}^{3+}$ concentration $\left(\mathrm{mg} \mathrm{L}^{-1}\right)$ & $1.52 \pm 0.32$ \\
$\mathrm{Zn}^{2+}$ concentration $\left(\mu \mathrm{g} \mathrm{L}^{-1}\right)$ & $98.5 \pm 3.3$ \\
\hline
\end{tabular}

\subsection{Membrane preparation}

The PIMs used in this research were prepared following the procedure described previously by us [16]. The cation-exchange extractant DNNS (35 wt $\%)$, the modifier 1-tetradecanol (10 wt $\%)$ and 
the base polymer PVC (55 wt\%) (total mass of $320.0 \mathrm{mg}$ ) were dissolved in $5.0 \mathrm{~mL}$ of THF under constant stirring. The clear solution was then poured into a glass ring ( $73 \mathrm{~mm}$ diameter), positioned on a flat glass plate. The glass ring was covered with filter paper and another glass plate to allow the slow evaporation of THF for a period of $24 \mathrm{~h}$, resulting in an oil-free, homogenous and transparent PIM. A maximum of 4 circular segments with a diameter of $25 \mathrm{~mm}$ were cut from the centre of each casted PIM, and used in the passive sampling experiments. The circular segments of PIMs with such diameter had an average mass of $22.3 \pm 2.5 \mathrm{mg}(\mathrm{n}=10)$ and an average thickness of $47.4 \pm 1.7 \mu \mathrm{m}(\mathrm{n}=6)$.

\subsection{Instrumentation}

A TPS SmartChem-Lab Multi-Parameter Laboratory Analyzer (Australia) was used to measure the $\mathrm{pH}$ and electrical conductivity of synthetic source solutions and the environmental freshwater sample.

The room temperature during each passive sampling experiment was monitored using a Humidity and Temperature USB Data Logger (Digitech), while the source solution temperature was monitored using a digital temperature probe (U-Lab Instruments, display resolution $<0.5^{\circ} \mathrm{C}$ ). A Motic SMZ-140 stereo microscope (Motic, China) with $60 \times$ magnification in combination with a MoticCam 1000 microscope camera (Motic, China) were used for the measurement of the thickness of the PIMs used in this study.

An atomic absorption spectrometer (AAS, Hitachi Z-2000 Series Polarized Zeeman, Japan) was used to determine the concentration of $\mathrm{Zn}^{2+}$ in both the source and receiving solutions used in most of the passive sampling experiments under the following instrument settings: lamp current $5 \mathrm{~mA}$, wavelength $213.9 \mathrm{~nm}$, slit $1.3 \mathrm{~nm}$, burner head: standard, burner height $7.5 \mathrm{~mm}$, flame: air- 
acetylene, oxidant gas pressure $160 \mathrm{kPa}$, fuel gas flow rate $1.8 \mathrm{~L} \mathrm{~min}^{-1}$. Under these conditions the limit of detection for $\mathrm{Zn}^{2+}$ was estimated as $1 \mu \mathrm{g} \mathrm{L}^{-1}$.

ICP-OES (Varian Vista Pro Axial, Varian, Australia) was used to measure the concentrations of elements such as $\mathrm{Na}, \mathrm{K}, \mathrm{Mg}, \mathrm{Ca}, \mathrm{Fe}$, and $\mathrm{Al}$ in the spiked freshwater samples used as source solutions and in the corresponding receiving solutions. The measurements were performed under the following instrument settings: power $1.3 \mathrm{~kW}$, plasma flow $18 \mathrm{~L} \mathrm{~min}^{-1}$, auxiliary flow $1.5 \mathrm{~L}$ $\min ^{-1}$, nebuliser flow $0.75 \mathrm{~L} \mathrm{~min}^{-1}$, wavelengths in $\mathrm{nm}$ : 589.6 for $\mathrm{Na}, 766.5$ for $\mathrm{K}, 285.2 \mathrm{for} \mathrm{Mg}$, 317.9 for $\mathrm{Ca}, 259.9$ for $\mathrm{Fe}$, and 308.2 for $\mathrm{Al}$.

A peristaltic pump (Miniplus 3, Gilson, USA) was used to propel the source solution at a desired flow rate in the optimization of the flow-through PSD conditions.

MP6 piezoelectric micro pumps (Bartels Mikrotechnik, Germany) driven by an mp6-quad OEM were used in the flow-through PSD experiments using environmental waters spiked with $\mathrm{Zn}^{2+}$ and were further investigated for their potential use in field-based applications. The Bartels Mikrotechnik MP6 micro pump is a miniature microfluidic device $(30 \times 15 \times 3.8 \mathrm{~mm}, 2 \mathrm{~g})$ with low power consumption $(50 \mathrm{~mW})$ based on a piezoelectric diaphragm that has passive check valves to control the flow direction. Voltage applied to a piezo-ceramic mounted on a coated brass membrane results in deformation, causing upstroke/downstroke motion that alternatively draws in/pushes out the liquid from the pump chamber. The range of liquid flow rates for this device is 0.1-7 $\mathrm{mL} \mathrm{min}^{-1}$, controllable by the drive amplitude and frequency. These micro pumps have a life time of $\sim 5000 \mathrm{~h}$. The MP6-Quad OEM is a small printed circuit board $(38 \times 18 \times 12 \mathrm{~mm})$ that allows up to four MP6 micropumps to be conveniently controlled by a microprocessor using an industry standard two wire $\mathrm{I}^{2} \mathrm{C}$ interface. The shape, frequency and amplitude of the MP6-Quad 
OEM output waveforms for each of the micro pumps are controlled by simply setting values in an internal register stack.

The custom MP6 controller developed for this work incorporates an MP6-Quad OEM and a propeller (Parallax Inc) 32 bit microprocessor. Although the MP6-Quad OEM is powered by only $3.3 \mathrm{~V}$ (drawing $75 \mathrm{~mA}$ with 4 pumps running at full output), it generates waveforms up to $250 \mathrm{~V}$ peak-to-peak when driving an MP6 to deliver its maximum flow rate of $\sim 7 \mathrm{~mL} \mathrm{~min}^{-1}$. In the experiments conducted as part of this study, an 8 bit frequency value was held fixed and the flow rate was then linear in the 5 bit (0-31) amplitude setting.

\subsection{Passive sampling devices}

In a previous study, a PIM-based passive sampler consisting of a glass vessel with screw threads on each end, a $25 \mathrm{~mm}$ screw cap with aperture, a $14 \mathrm{~mm}$ screw cap without aperture, a Teflon washer, and a rubber o-ring was developed for passive sampling of ammonia in freshwaters [16]. The glass vessels were made by joining together two screw thread glass tubes with different diameters (i.e., 24 and $14 \mathrm{~mm}$ ) to create a $5.4 \mathrm{~cm}$ long bottle-shaped container that accommodated $10 \mathrm{~mL}$ of receiving solution. This device was used in the present work to examine the effect of different flow patterns on the source solution, and it is referred to throughout this manuscript as a dip-in passive sampler.

In the present study, a new flow-through PSD (Figure 1a) was developed which consisted of a 3Dprinted flow-through compartment (Figures $1 \mathrm{~b}$ to $1 \mathrm{c}$ ) and the dip-in PSD mentioned above excluding the 24-mm cap with aperture. The new 3D-printed flow-through compartment was designed using Solidworks student edition 2015 - 2016 and printed using Object Eden260VS

(Stratasys, USA) or UP PLUS2 3D printers (3D Printing System, Australia). Two different 3D- 
printed filament types were used in this research: $1.75 \mathrm{~mm}$ acrylonitrile butadiene styrene (ABS) plastic filament (Figure 2a) for printing using the UP PLUS2 3D printer and VeroClear-RGD810 filament (transparent poly(methyl methacrylate) or acrylic, Figure $2 \mathrm{~b}$ ) for printing using the Object Eden260VS 3D printer.

The 3D-printed flow-through compartment comprised three threaded holes with an internal diameter of $23 \mathrm{~mm}$ so as to fit the 24-mm screw ends of 3 glass vessels (Figure $1 \mathrm{~b}$ and $1 \mathrm{~d}$ ) thus allowing each experiment to be conducted in triplicate. A $5 \mathrm{~mm}$ deep well with an internal diameter of $14 \mathrm{~mm}$, placed below each one of the $23 \mathrm{~mm}$ threaded holes, was created to allow the flowing source solution to be in contact with the whole surface of the PIM covering the corresponding glass vessel (Figure 1c). In order for the source solution to flow in and out of each well, a $2.28 \mathrm{~mm}$ diameter channel was created.

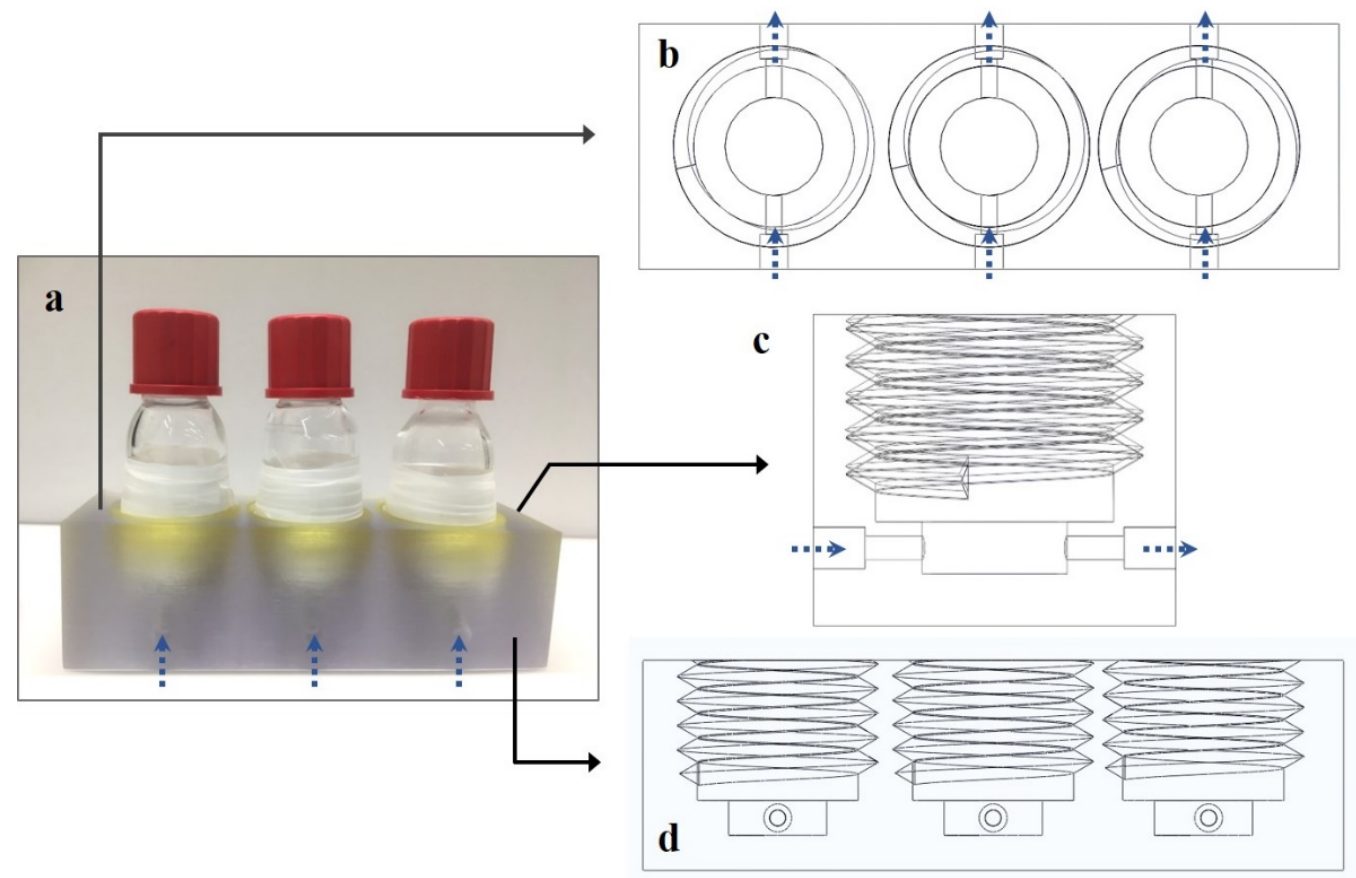

Figure 1. Photographic image of the flow-through PSD (a) and schematic representation of the 3D-printed flow-through passive sampling compartment: top (b), side (c) and front (d) views. Dashed arrows indicate the flow direction of the source solution. 

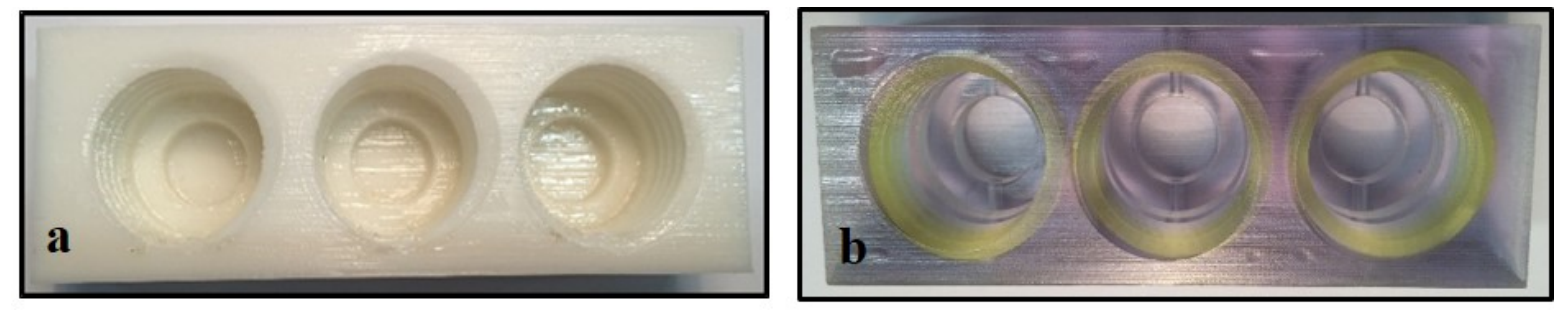

Figure 2. Flow-through passive sampling compartment 3D-printed using the acrylonitrile butadiene styrene (ABS) filament (a) or the acrylic filament (b).

\subsection{Passive sampling experiments}

\subsubsection{Experiments using the flow-through PSDs}

The flow-through PSDs were assembled by incorporating a $25 \mathrm{~mm}$ DNNS-based PIM placed between a Teflon washer (Gasketech, Australia) and a rubber o-ring (Reece, Australia) which were positioned to sit on top of the $5 \mathrm{~mm}$ deep well of the 3D-printed flow-through device. The thread of the glass vessel was wrapped with Teflon tape (Reece, Australia) to create a waterproof seal and it was then closely screwed into the threaded hole of the 3D-printed flow-through compartment. After the PIM had been securely placed, $10.0 \mathrm{~mL}$ of $1.0 \mathrm{~mol} \mathrm{~L}^{-1} \mathrm{HNO}_{3}$ receiving solution were pipetted into the glass vessel. To allow the transport of source solution through the 3D-printed flow-through device, each channel was connected to one of the channels of a 4-channel peristaltic pump (Minipuls 3, Gilson, USA) via a male luer slip to 200 series barbs (1/16 in ID tubing white nylon, Chromalytic, Australia), pump tubing (Tygon tubing, $1.14 \mathrm{~mm}$ ID, TACS, Australia) and Teflon tubing ( $0.8 \mathrm{~mm}$ ID, Supelco, USA). A peristaltic pump circulated the source solution at a desired flow rate from its container, through the wells, into the waste container. The passive sampling experiments using spiked freshwater were conducted using MP6 piezoelectric pumps driven by an MP6 Quad OEM controller (Bartels Mikroteknik, Germany). A micro filter 
(MP-filter, $21 \times 5.5 \mathrm{~mm}$ size, $20-60 \mu \mathrm{m}$ porosity) (Bartels Mikroteknik, Germany) was placed at the pump inlet to filter any fine particulates present in the spiked freshwater used as the source solution.

In all experiments using the newly developed flow-through PSDs, the source solution was not recirculated (Figure 3a), except for the experiments where the effect of water turbulence on the accumulation of $\mathrm{Zn}^{2+}$ by the flow-through PSDs (Figure 3b) was assessed. In this case, the effluent source solution was returned to the original source solution container and recirculated during a 5day experiment.
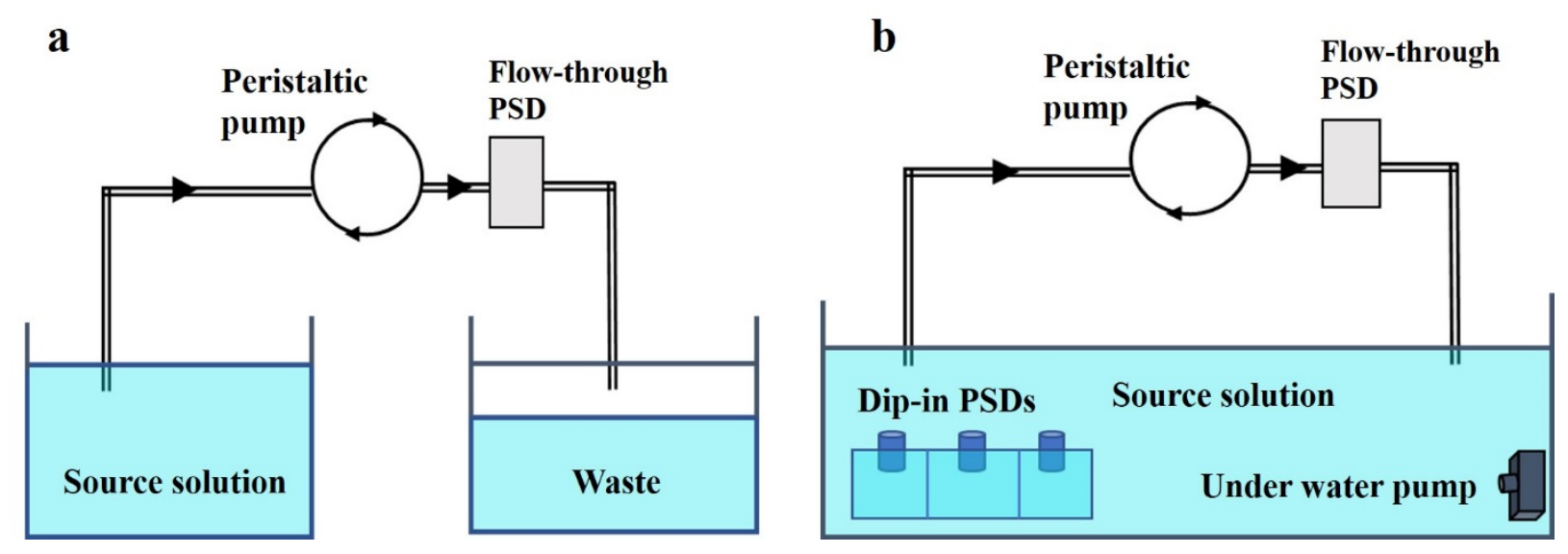

Figure 3. Schematic representation of the preliminary passive sampling experimental set-up incorporating the flow-through PSD (a) and the passive sampling experimental set-up used to examine the effect of source solution flow pattern on flow-through PSD, dip-in PSD and shielded dip-in PSD (b).

\subsubsection{Experiments using the dip-in and shielded dip-in PSDs}

In a previous study, we developed an approach to minimize the effect of the flow pattern on dipin passive samplers (Figure $4 \mathrm{a}$ ) by using a $4 \mathrm{~cm}$ long plastic cylindrical-shaped barrier. These samplers are referred to as shielded dip-in passive samplers (Figure 4b) since the cylindrical- 
shaped barrier creates a stagnant liquid diffusion layer at the PIM/source solution interface thus shielding the PIM from the actual flow pattern of the aquatic system. This approach was successfully proven to minimize the effect of the flow pattern on the passive sampling of antibiotics [19]. It was of interest in the current study to examine the effect of the flow pattern on the accumulation of $\mathrm{Zn}^{2+}$ when using the newly developed flow-through passive samplers and to compare these results to those using the previously developed PIM-based passive samplers, including the dip-in and shielded dip-in samplers. In the corresponding experiments a newly developed PSD was immersed in a $15 \mathrm{~L}$ container containing $10.0 \mathrm{~L}$ of synthetic source solution together with a dip-in PSD and a shielded dip-in PSD (Figure 4) which were operated as described previously [16]. The 3 PSDs in each container were positioned on a polypropylene test tube rack (Kartell, 24 place, $30 \mathrm{~mm}$ wide) with the PIM-end of the glass vessels facing down.

Each of the passive samplers used was assembled by incorporating a circular segment of DNNSbased PIM in between the Teflon washer and an o-ring in the $25 \mathrm{~mm}$ cap with aperture. The glass vessel, previously wrapped with Teflon tape on its thread, was closely screwed into the same cap. Then, $10.0 \mathrm{~mL}$ of $1.0 \mathrm{~mol} \mathrm{~L}^{-1} \mathrm{HNO}_{3}$ solution (used as the receiving solution) was transferred into each sampler.
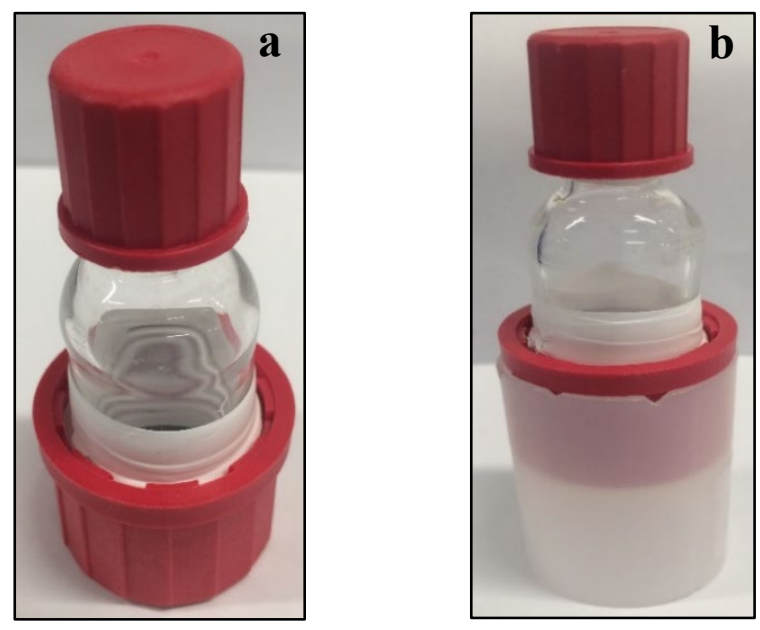

Figure 4. Photographs of a dip-in passive sampler (a) and a shielded dip-in passive sampler (b). 
In addition to an experiment using a stagnant source solution, two techniques were used to create different source solution flow patterns: (a) circulation using an underwater pump immersed inside the source solution (AQUAPRO low voltage tabletop feature pump, Aquatec Equipment, China) and (b) circulation promoted by placing the source solution container on top of an orbital shaker rotated at $45 \mathrm{rpm}$.

Each passive sampling experiment was conducted for 5 consecutive days and $0.500 \mathrm{~mL}$ of the receiving solution was collected every day; this was replaced with the same volume of the original receiving solution. The collected samples were then diluted to $2.50 \mathrm{~mL}$ by the addition of $5.0 \%$ $\mathrm{HNO}_{3}$ solution, and the $\mathrm{Zn}^{2+}$ concentration was determined using AAS. The effect of dilution due to the sample collection and replacement was taken into account when processing the data. A schematic diagram of all the passive sampling experiments is shown in Figure 3.

\section{Results and discussion}

\subsection{PIM extraction and transport of $\mathrm{Zn}^{2+}$}

A PIM containing $35 \mathrm{wt} \%$ commercial DNNS, $55 \mathrm{wt} \%$ PVC and $10 \mathrm{wt} \%$ 1-tetradecanol has been previously used by us as a suitable semipermeable membrane in the passive sampling of the ammonium cation [16]. In another study involving $\mathrm{Zn}^{2+}$ PIM-based passive sampling, it has been demonstrated that $0.10 \mathrm{~mol} \mathrm{~L}^{-1} \mathrm{HNO}_{3}$ solution is suitable as a receiving solution [15]. In the present study, $1.0 \mathrm{~mol} \mathrm{~L}^{-1} \mathrm{HNO}_{3}$ was chosen as the receiving solution with the intention of increasing the $\mathrm{Zn}^{2+}$ accumulation capacity of the newly developed flow-through PSD.

When the passive samplers were exposed to the source solution, the DNNS-based PIMs acted as the semipermeable membranes separating the $1.0 \mathrm{~mol} \mathrm{~L}^{-1} \mathrm{HNO}_{3}$ receiving solution from the 
flowing source solution. $\mathrm{Zn}^{2+}$ ions in the source solution were extracted into the PIM by forming ion pairs with the deprotonated DNNS anions (Eq. 1) [31].

$$
\mathrm{Zn}^{2+}{ }_{(\mathrm{aq})}+2 \mathrm{HD}{ }_{(\mathrm{PIM})} \rightleftarrows \mathrm{ZnD}_{2}(\mathrm{PIM})+2 \mathrm{H}^{+}{ }_{(\text {aq })}
$$

where the subscripts $(a q)$ and (PIM) refer to the aqueous source solution and the membrane, respectively, and $H D$ corresponds to the extractant DNNS.

These ions-pairs then diffused across the PIM towards the PIM/receiving solution interface where the $\mathrm{Zn}^{2+}$ ions were stripped into the strongly acidic receiving solution by protonating the corresponding DNNS anions.

The acidity of the receiving solution drives the transport of $\mathrm{Zn}^{2+}$, so transport will occur as long as the acidity of the receiving solution is higher than that of the source solution.

\subsection{Performance of the 3D-printed flow-through PSD}

Passive sampling experiments were conducted for 5 days to compare the performance of two different materials used for the 3D-printing of the flow-through compartments of the device. The source solution contained only $100.0 \mu \mathrm{g} \mathrm{L}^{-1} \mathrm{Zn}^{2+}$ in deionized water and $10.0 \mathrm{~mL}$ of $1.0 \mathrm{~mol} \mathrm{~L}^{-1}$ $\mathrm{HNO}_{3}$ was used as the receiving solution. The source solution was continuously pumped through the flow-through PSDs at $0.50 \mathrm{~mL} \mathrm{~min}{ }^{-1}$ for 5 days using a peristaltic pump and the amount of $\mathrm{Zn}^{2+}$ accumulated in the receiving solution was monitored daily (Figure $3 \mathrm{a}$ ). There was a linear uptake of $\mathrm{Zn}^{2+}$ regardless of the material used to 3D print the corresponding flow-through compartments (Figure 5). However, the amount of $\mathrm{Zn}^{2+}$ accumulated in the receiving solution of the flow-through PSD printed with acrylic was two times higher than that for the one printed using ABS filament. Air bubbles were observed inside the well of the ABS 3D-printed flow-through device during the experiments, which most likely affected $\mathrm{Zn}^{2+}$ accumulation by reducing the 
surface area of the PIM in contact with the source solution. The ABS 3D-printed flow-through compartments had a porous structure which allowed air to diffuse into the flowing source solution where it formed bubbles. A treatment to seal the pores on the surface of the ABS 3D-printed flowthrough compartments was attempted unsuccessfully by using acetone vapor. It was expected that this vapor was going to initially dissolve the porous surface layer of the compartments resulting in the formation of a surface dense layer.

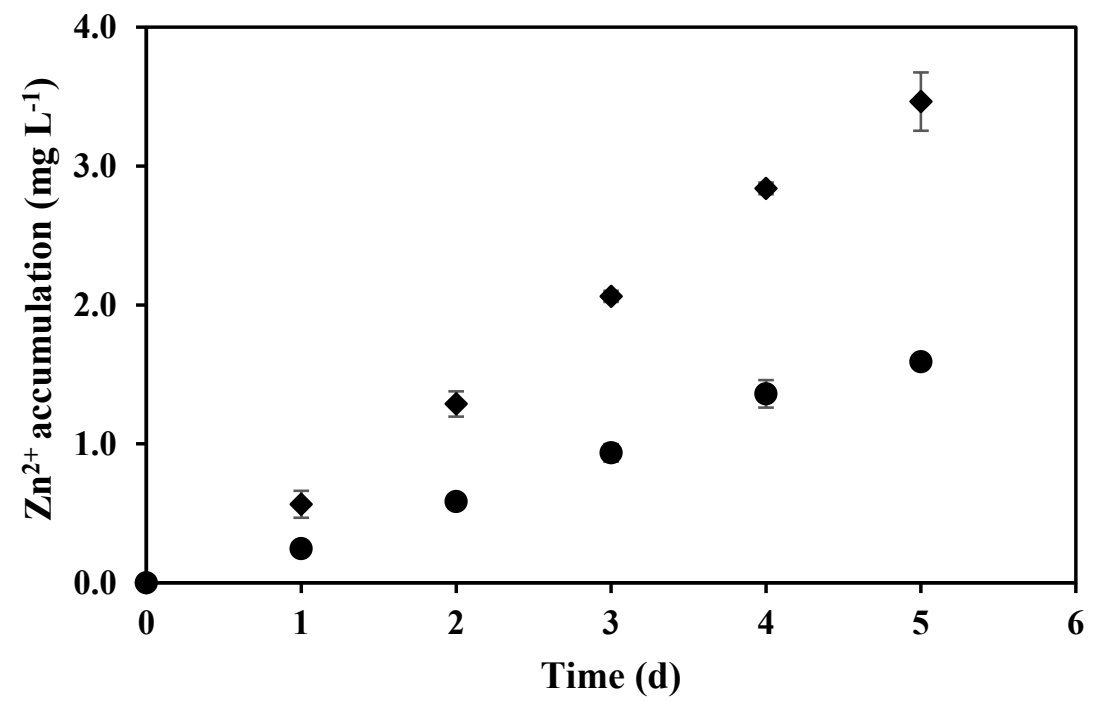

Figure 5. $\mathrm{Zn}^{2+}$ accumulation in the receiving solutions of flow-through PSDs printed with acrylonitrile butadiene styrene $(\bullet)$ or poly(methyl methacrylate) $(\bullet)$. Experimental conditions: Source solution: $100.0 \mu \mathrm{g} \mathrm{L}-1 \mathrm{Zn}^{2+}$, receiving solution: $10.0 \mathrm{~mL}$ of $1.0 \mathrm{~mol} \mathrm{~L}^{-1} \mathrm{HNO}_{3}$, peristaltic pump flow rate: $0.50 \mathrm{~mL} \mathrm{~min}{ }^{-1}$, PIM composition: $35 \mathrm{wt} \%$ DNNS, $55 \mathrm{wt} \%$ PVC and $10 \mathrm{wt} \% 1$ tetradecanol, sampling period: 5 days. Experiments were conducted in triplicate. Error bars are \pm standard deviation (SD) for $1 \sigma_{\mathrm{n}-1}(\mathrm{n}=3)$. 
This problem with air bubbles, on the other hand, was not encountered when using the acrylic 3Dprinted flow-through devices. Therefore, it was decided to use the acrylic material to $3 \mathrm{D}$ print the flow-through compartments for use in further passive sampling experiments.

\subsection{Effect of the source solution flow rate}

The source solution flow rate was expected to influence the transport of $\mathrm{Zn}^{2+}$ across the PIM into the receiving solution. Therefore, experiments were conducted where the source solution flow rate was varied between 0.2 and $3 \mathrm{~mL} \mathrm{~min}^{-1}$ to find its optimum value in terms of $\mathrm{Zn}^{2+}$ accumulation in the receiving solution (Figure 6).

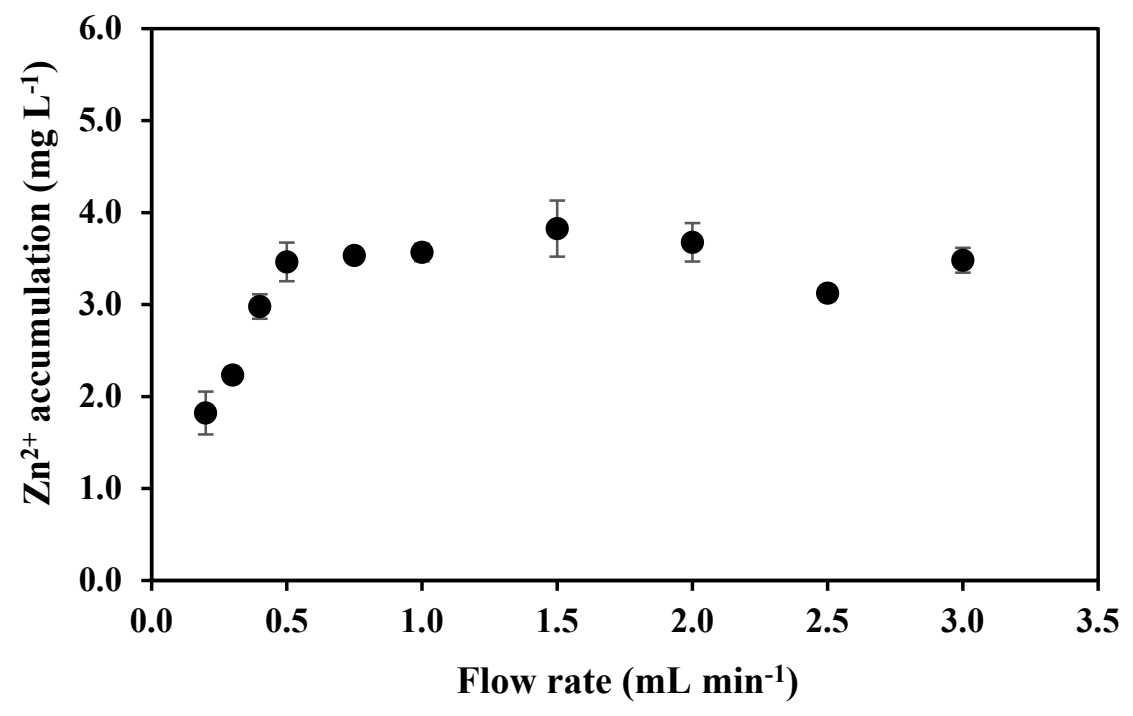

Figure 6. Influence of the source solution flow rate on the accumulation of $\mathrm{Zn}^{2+}$ into the receiving solution of the flow-through PSDs over a 5-day period. Experimental conditions as in Figure 5. Error bars are $\pm \mathrm{SD}$ for $1 \sigma_{\mathrm{n}-1}(\mathrm{n}=3)$.

The results obtained suggest that at flow rates lower than $0.50 \mathrm{~mL} \mathrm{~min}{ }^{-1}$, the amount of $\mathrm{Zn}^{2+}$ accumulated by the flow-through PSDs increased as the flow rate increased. This was most 
probably due to the increase of the volume of source solution being in contact with the membrane and the decrease of the stagnant diffusion layer thickness, which led to more $\mathrm{Zn}^{2+}$ being transported through the PIM to the receiving solution. However, at flow rates $>0.50 \mathrm{~mL} \mathrm{~min}{ }^{-1}, \mathrm{Zn}^{2+}$ accumulation plateaued. This could be explained by the fact that at these relatively high flow rates the $\mathrm{Zn}^{2+}$ mass transfer rate across the stagnant diffusion layer was equal to the maximum $\mathrm{Zn}^{2+}$ mass transfer rate across the PIM which was independent of the source solution flow rate. Thus, a flow rate of $0.50 \mathrm{~mL} \mathrm{~min}{ }^{-1}$ was chosen for the subsequnet experiments with the flow-through PSDs.

\subsection{Effect of the source solution flow pattern}

The flow pattern in aquatic systems is known to be one of the main environmental factors that significantly affect the reliability of passive sampling. The main reason for this is that it controls the thickness of the stagnant diffusion layer at the membrane/source solution interface which determines to a great extent the overall rate of the target analyte transport from the source solution to the receiving solution [32]. Hence, it was important to evaluate the effect of different flow patterns on the accumulation of $\mathrm{Zn}^{2+}$ by the newly developed flow-through PSD and compare these results with those for the previously developed dip-in [15] and shielded dip-in passive samplers [19]. Three different source solution flow patterns were created, namely stagnant water, turbulence induced by an AQUAPRO low voltage tabletop feature pump set at a flow rate of $200 \mathrm{~L} \mathrm{~h}^{-1}$ and a circular flow pattern promoted by an orbital shaker set at shaking rate of $45 \mathrm{rpm}$. The corresponding experiments were conducted in triplicate for 5 consecutive days under the three flow patterns studied and the results, expressed as amount of $\mathrm{Zn}^{2+}$ accumulated, are shown in Figure 7. 


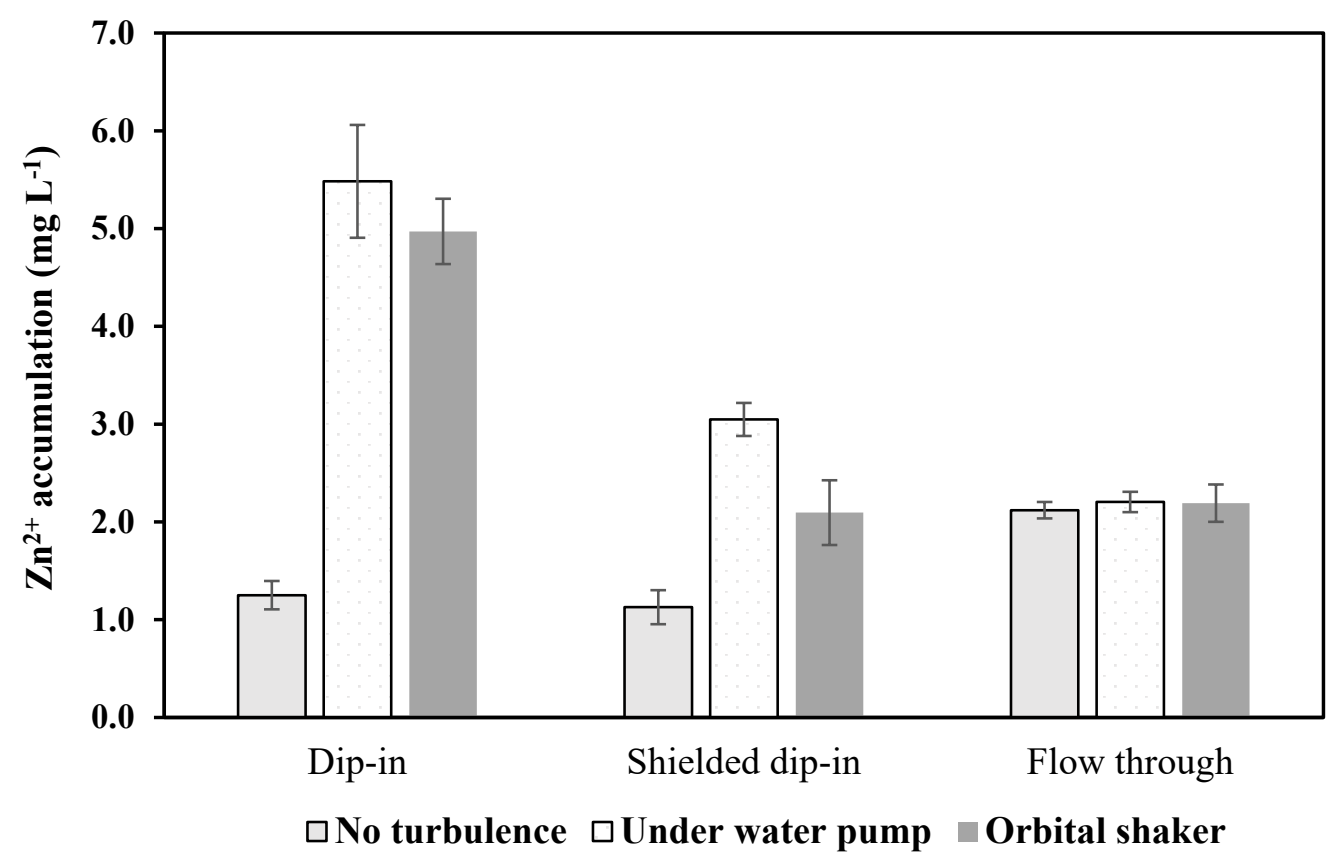

Figure 7. Effect of source solution flow pattern on the accumulation of $\mathrm{Zn}^{2+}$ in the receiving solution of the dip-in, shielded dip-in and flow-through PSDs over a 5-day period. Experimental conditions as in Figure 5. Error bars are $\pm \mathrm{SD}$ for $1 \sigma_{\mathrm{n}-1}(\mathrm{n}=3)$.

The results show that the source solution flow pattern affected significantly the amount of $\mathrm{Zn}^{2+}$ accumulated by the dip-in and shielded dip-in passive samplers. The use of a cylindrical-shaped shield in the shielded dip-in passive sampler device reduced to a considerable extent the flow pattern effects in accordance with the results for this type of passive sampler reported by Garcia et al. [19]. The results presented in Figure 6 clearly show that the source solution flow patterns had no effect on the accumulation of $\mathrm{Zn}^{2+}$ in the case of the newly developed flow-through PSDs.

\subsection{Miniaturization of the flow-through PSD - suitability of piezoelectric micro pumps}

Peristaltic pumps are not suitable propelling devices for field applications of the PSD developed 
as part of this study. Therefore, the suitability of piezoelectric micro pumps was explored and their performance was compared to the performance of conventional peristaltic pumps.

The pumping performance of three micro pumps and the three channels of a peristaltic pump was monitored over a 30-day period, corresponding to 6 consecutive 5-day passive sampling experiments. The pump settings were adjusted to ensure initial average flow rates of approximately $0.50 \mathrm{~mL} \mathrm{~min}^{-1}$. Each flow rate was determined once daily by weighing the amount of deionized water pumped over a period of $5 \mathrm{~min}$.

The piezoelectric micro pumps delivered highly reproducible long-term performance, more so than the peristaltic pump (Figure 8 ). An average flow rate decrease of $\sim 15 \%$ over the 30 -day period was observed when the peristaltic pumps were used which was due to gradual stretching of the Tygon pump tubing over time while the average flow rate decrease for the piezoelectric micro pumps was only $\sim 2.5 \%$. Therefore, it was concluded that the piezoelectric pumps studied were suitable propelling devices for the newly developed flow-through PSD.

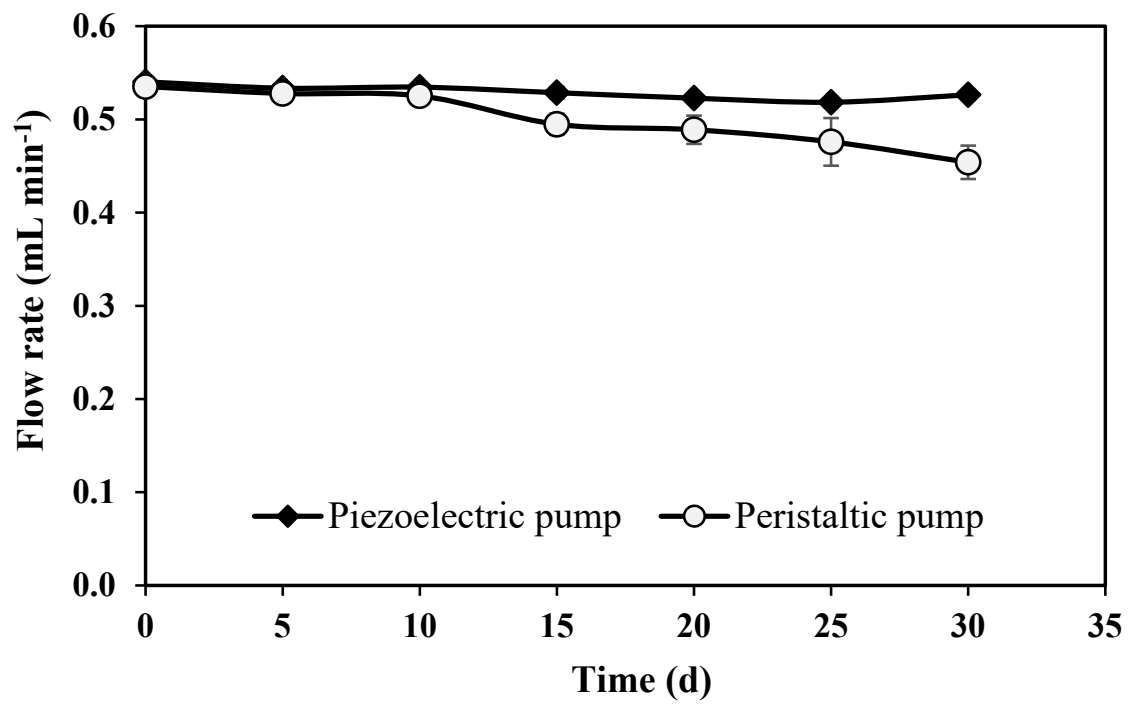

Figure 8. The average flow rate of the piezoelectric micro pumps $(\diamond)$ and the three channels of the peristaltic pump $(\mathrm{O})$ in the case of continuous pumping over a 30-day period consisting of 6 consecutive 5-day cycles. Error bars are $\pm \operatorname{SD}$ for $1 \sigma_{n-1}(n=3)$. 
The effect of the backpressure on the flow rate, arising from the use of micro filters, was also investigated by comparing the average flow rates of piezoelectric micro pumps and peristaltic pumps with and without micro filters. The results for both pump configurations with and without micro filters were statistically indistinguishable indicating the absence of a backpressure effect. This has the operational advantage that no adjustment of pump settings is required when using a micro filter.

\subsection{Laboratory testing of the flow-through PSD}

To test the performance of the newly developed flow-through PSD using the piezoelectric micro pumps, a laboratory-based experiment with environmental freshwater spiked with $100.0 \mu \mathrm{g} \mathrm{L}^{-1}$ $\mathrm{Zn}^{2+}$ as the source solution was conducted. The same experiment was conducted in parallel using a peristaltic pump. In these experiments, both types of pumps had a micro filter at the inlet to prevent the tubing and the micro pumps' chamber from blocking by fine particles that might be present in the spiked environmental water. The source solution container was continuously shaken during this experiment by an orbital shaker at 45 rpm shaking rate to create a random flow pattern to mimic environmental conditions.

The average concentration of $\mathrm{Zn}^{2+}$ in the receiving solution on day 5 was $0.752 \pm 0.048 \mathrm{mg} \mathrm{L}^{-1}$ and $0.756 \pm 0.025 \mathrm{mg} \mathrm{L}^{-1}$ in the case of using piezoelectric micro pumps and a peristaltic pump, respectively (Figure 9). The average chemical composition of the respective receiving solutions is shown in Table 2. These results for the two types of pumps were similar and there was no statistically significant difference in the average amounts of $\mathrm{Zn}^{2+}$ accumulated in the corresponding receiving solutions at the $95 \%$ confidence level (2-tail Student's $t$-test: $0.127<$ 2.57). Moreover, no blockage of either the peristaltic pump tubing or the piezoelectric micro 
pumps' chambers was observed after the 5-day experiment since the flow rates of the source solution remained unchanged. These findings further confirmed the suitability of the piezoelectric micro pumps as portable and inexpensive propelling devices for the newly developed flow-through PSD. It is also important to note that no signs of biofouling were observed on the surface of the PIMs at the end of the experiments which confirmed that the highly acidic DNNS-based PIMs were able to prevent the growth of microorganisms on their surface.

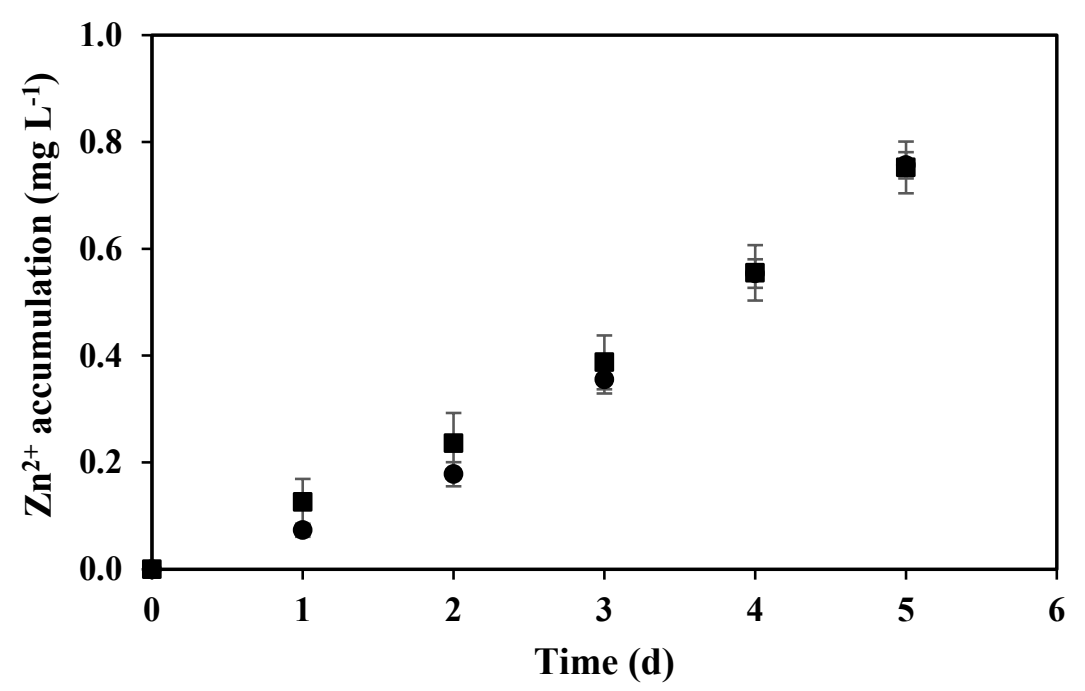

Figure 9. Average transient $\mathrm{Zn}^{2+}$ concentration in the receiving solutions in flow-through passive sampling experiments with environmental freshwater spiked with $100.0 \mu \mathrm{g} \mathrm{L} \mathrm{L}^{-1} \mathrm{Zn}^{2+}$ when piezoelectric micro pumps ( $(\mathbf{)})$ or the three channels of a peristaltic pump $(\bullet)$ were used. Remaining experimental conditions as in Figure 5. Error bars are \pm standard deviation for $1 \sigma_{\mathrm{n}-1}(\mathrm{n}=3)$. 
Table 2. Chemical composition of the receiving solutions of the flow-through PDSs using peristaltic or micro piezoelectric pumps. The source solution consisted of environmental freshwater spiked with $100.0 \mu \mathrm{g} \mathrm{L}^{-1} \mathrm{Zn}^{2+}$.

\begin{tabular}{cll}
\hline \multirow{3}{*}{ Elements } & \multicolumn{2}{l}{$\mathrm{Zn}^{2+}$ concentration in the receiving solution $\left(\mathrm{mg} \mathrm{L}^{-1}\right)$} \\
\cline { 2 - 3 } & PSD using peristaltic pump & PSD using micro piezoelectric pumps \\
\hline $\mathrm{Na}$ & $88.4 \pm 17.1$ & $84.8 \pm 24.9$ \\
$\mathrm{~K}$ & $18.3 \pm 2.1$ & $16.0 \pm 3.2$ \\
$\mathrm{Mg}$ & $103 \pm 4$ & $90.9 \pm 14.6$ \\
$\mathrm{Ca}$ & $81.6 \pm 3.7$ & $69.5 \pm 12.1$ \\
$\mathrm{Fe}$ & $0.024 \pm 0.018$ & $0.033 \pm 0.025$ \\
$\mathrm{Al}$ & $0.008 \pm 0.004$ & $0.045 \pm 0.019$ \\
$\mathrm{Zn}$ & $0.756 \pm 0.024$ & $0.752 \pm 0.054$ \\
\hline
\end{tabular}

\section{Conclusions}

A compact, inexpensive and portable flow-through passive sampler for $\mathrm{Zn}^{2+}$ which is not influenced by the flow pattern of the sampled aquatic system, has been developed and tested successfully under laboratory conditions. It uses a PIM, composed of PVC, DNNS and 1tetradecanol, as its semipermeable barrier separating the receiving solution in a glass container from the sampled aquatic system, and a 3D-printed polymeric flow-through section. The determination of the $\mathrm{Zn}^{2+}$ concentration, accumulated during the sampling period in the receiving solution, can be performed directly without laborious and time-consuming pre-treatment steps normally encountered in passive sampling of metal ions using solid receiving phases.

The optimal flow rate of the source solution through the passive sampler has been found to be 0.50 $\mathrm{mL} \min ^{-1}$. It has been demonstrated that the acrylic polymer is more suitable for the $3 \mathrm{D}$ printing of the device compared to ABS polymer. The suitability of inexpensive piezoelectric micro pumps, 
equipped with micro filters to prevent blockage, as the propelling devices for the newly developed flow-through passive sampler has been established in a 30-day experiment. The complete passive sampling system is $12 \times 12 \times 7 \mathrm{~cm}$ and costs about $100 €$. It has an energy consumption of $50 \mathrm{~mW}$ $(15 \mathrm{~mA} \times 3.3 \mathrm{~V})$, and can be powered by a small rechargeable lithium battery $(69 \times 19 \mathrm{~mm}, 47.5$

g) for at least 5 days. Moreover, the PIM used has been shown to be completely resistant to biofouling in 5-day passive sampling experiments with environmental water spiked with $\mathrm{Zn}^{2+}$.

On the basis of the results obtained it can be concluded that the newly developed PSD offers the opportunity for inexpensive passive sampling of environmental waters which is independent of flow pattern and biofouling effects.

\section{Acknowledgements}

The authors would like to thank the Australian Research Council (ARC) and Melbourne Water Corporation for funding this research (grant LP160100687). Fidelis Nitti acknowledges Indonesian Endowment Fund for Education (LPDP) for providing the PhD scholarship. 


\section{References}

[1] B. Vrana, G.A. Mills, I.J. Allan, E. Dominiak, K. Svensson, J. Knutsson, G. Morrison, R. Greenwood, Passive sampling techniques for monitoring pollutants in water, Trends in Analytical Chemistry, 24 (2005) 845-868.

[2] T. Gorecki, J. Namiesnik, Passive sampling, Trends in Analytical Chemistry, 21 (2002) 276291.

[3] A. Kot, B. Zabiegala, J. Namiesnik, Passive sampling for long-term monitoring of organic pollutants in water, Trends in Analytical Chemistry, 19 (2000) 446-459.

[4] A. Kot-Wasik, B. Zabiegala, M. Urbanowicz, E. Dominiak, A. Wasik, J. Namiesnik, Advances in passive sampling in environmental studies, Analytica Chimica Acta, 602 (2007) $141-163$.

[5] J. Namiesnik, B. Zabiegala, A. Kot-Wasik, M. Partyka, A. Wasik, Passive sampling and/or extraction techniques in environmental analysis: a review, Analytical and Bioanalytical Chemistry, 381 (2005) 279-301.

[6] S. Seethapathy, T. Gorecki, X. Li, Passive sampling in environmental analysis, Journal of Chromatography A, 1184 (2008) 234-253.

[7] I.J. Allan, J. Knutsson, N. Guigues, G.A. Mills, A.M. Fouillac, R. Greenwood, Evaluation of the Chemcatcher and DGT passive samplers for monitoring metals with highly fluctuating water concentrations, Journal of Environmental Monitoring, 9 (2007) 672-681.

[8] I.J. Allan, J. Knutsson, N. Guigues, G.A. Mills, A.M. Fouillac, R. Greenwood, Chemcatcher ${ }^{(\mathrm{R})}$ and DGT passive sampling devices for regulatory monitoring of trace metals in surface water, Journal of Environmental Monitoring, 10 (2008) 821-829. 
[9] A. Charriau, S. Lissalde, G. Poulier, N. Mazzella, R. Buzier, G. Guibaud, Overview of the Chemcatcher ${ }^{(\mathrm{R})}$ for the passive sampling of various pollutants in aquatic environments Part A: Principles, calibration, preparation and analysis of the sampler, Talanta, 148 (2016) 556571.

[10] W. Davison, H. Zhang, In situ speciation measurements of trace components in natural waters using thin-film gels, Nature, 367 (1994) 546-548.

[11] R. Romero, J.Å. Jönsson, Determination of free copper concentrations in natural waters by using supported liquid membrane extraction under equilibrium conditions, Analytical and Bioanalytical Chemistry, 381 (2005) 1452-1459.

[12] V.I. Slaveykova, N. Parthasarathy, J. Buffle, K.J. Wilkinson, Permeation liquid membrane as a tool for monitoring bioavailable $\mathrm{Pb}$ in natural waters, Science of the Total Environment, 328 (2004) 55-68.

[13] W.G. Brumbaugh, J.D. Petyy, J.N. Huckins, S.E. Manahan, Stabilized Liquid Membrane Device (SLMD) for the passive, integrative sampling of labile metals in water, Water, Air, and Soil Pollution, 133 (2002) 109-119.

[14] L. Chimuka, E. Cukrowska, J.A. Jonsson, Why liquid membrane extraction is an attractive alternative in sample preparation, Pure and Applied Chemistry, 76 (2004) 707-722.

[15] M.I.G.S. Almeida, C. Chan, V.J. Pettigrove, R.W. Cattrall, S.D. Kolev, Development of a passive sampler for Zinc(II) in urban pond waters using a polymer inclusion membrane, Environmental Pollution, 193 (2014) 233-239.

[16] M.I.G.S. Almeida, A.M. Silva, R.A. Coleman, V.J. Pettigrove, R.W. Cattrall, S.D. Kolev, Development of a passive sampler based on a polymer inclusion membrane for total 
ammonia monitoring in freshwaters, Analytical and Bioanalytical Chemistry, 408 (2016) $3213-3222$.

[17] M.I.G.S. Almeida, R.W. Cattrall, S.D. Kolev, Recent trends in extraction and transport of metal ions using polymer inclusion membranes (PIMs), Journal of Membrane Science, 415416 (2012) 9-23.

[18] L.D. Nghiem, P. Mornane, I.D. Potter, J. Perera, R.W. Cattrall, S.D. Kolev, Extraction and transport of metal ions and small organic compounds using polymer inclusion membranes (PIMs), Journal of Membrane Science, 281 (2006) 7-41.

[19] A. Garcia-Rodríguez, C. Fontàs, V. Matamoros, M.I.G.S. Almeida, R.W. Cattrall, S.D. Kolev, Development of a polymer inclusion membrane-based passive sampler for monitoring of sulfamethoxazole in natural waters. Minimizing the effect of the flow pattern of the aquatic system, Microchemical Journal, 124 (2016) 175-180.

[20] J. Gimpel, H. Zhang, W. Hutchinson, W. Davison, Effect of solution composition, flow and deployment time on the measurement of trace metals by the diffusive gradient in thin films technique, Analytica Chimica Acta, 448 (2001) 93-103.

[21] Ø.A. Garmo, K.R. Naqvi, O. Røyset, E. Steinnes, Estimation of diffusive boundary layer thickness in studies involving diffusive gradients in thin films (DGT), Analytical and Bioanalytical Chemistry, 386 (2006) 2233-2237.

[22] I.B. Roll, R.U. Halden, Critical review of factors governing data quality of integrative samplers employed in environmental water monitoring, Water Research, 94 (2016) 200-207.

[23] K. Booij, F. Smedes, E.M. van Weerlee, Spiking of performance reference compounds in low density polyethylene and silicone passive water samplers, Chemosphere, 46 (2002) 1157-1161. 
[24] H.H. Liu, C.S. Wong, E.Y. Zeng, Recognizing the limitations of performance reference compound (PRC)-calibration technique in passive water sampling, Environmental Science \& Technology, 47 (2013) 10104-10105.

[25] V. Fauvelle, S.L. Kaserzon, N. Montero, S. Lissalde, I.J. Allan, G. Mills, N. Mazzella, J.F. Mueller, K. Booij, Dealing with flow effects on the uptake of polar compounds by passive samplers, Environmental Science \& technology, 51 (2017) 2536-2537.

[26] D.S. O'Brien, B. Chiswell, J.F. Mueller, A novel method for the in situ calibration of flow effects on a phosphate passive sampler, Journal of Environmental Monitoring, 11 (2009) $212-219$.

[27] J. Llorca, C. Gutierrez, E. Capilla, R. Tortajada, L. Sanjuan, A. Fuentes, I. Valor, Constantly stirred sorbent and continuous flow integrative sampler New integrative samplers for the time weighted average water monitoring, Journal of Chromatography A, 1216 (2009) 57835792.

[28] J. Petersen, D. Profrock, A. Paschke, J.A.C. Broekaert, A. Prange, Development and field test of a mobile continuous flow system utilizing Chemcatcher for monitoring of rare earth elements in marine environments, Environ. Sci.-Wat. Res. Technol., 2 (2016) 146-153.

[29] G. Allinson, P. Zhang, A. Bui, M. Allinson, G. Rose, S. Marshall, V. Pettigrove, Pesticide and trace metal occurrence and aquatic benchmark exceedances in surface waters and sediments of urban wetlands and retention ponds in Melbourne, Australia, Environmental Science and Pollution Research, 22 (2015) 10214-10226.

[30] M. Allinson, P. Zhang, A. Bui, J.H. Myers, V. Pettigrove, G. Rose, S.A. Salzman, R. Walters, G. Allinson, Herbicides and trace metals in urban waters in Melbourne, Australia 
(2011-12): concentrations and potential impact, Environmental Science and Pollution Research, 24 (2017) 7274-7284.

[31] M. Ershad, M.I.G.S. Almeida, T.G. Spassov, R.W. Cattrall, S.D. Kolev, Polymer inclusion membranes (PIMs) containing purified dinonylnaphthalene sulfonic acid (DNNS): Performance and selectivity, Separation and Purification Technology, 195 (2018) 446-452.

[32] Z. Křesinová, K. Petrů, O. Lhotský, T. Rodsand, T. Cajthaml, Passive sampling of pharmaceuticals and personal care products in aquatic environments, European Journal of Environmental Sciences, 6 (2016) 43-56. 


\section{University Library}

\section{- M M N E R VA A gateway to Melbourne's research publications}

Minerva Access is the Institutional Repository of The University of Melbourne

Author/s:

Nitti, F;Almeida, MIGS;Morrison, R;Cattrall, RW;Pettigrove, VJ;Coleman, RA;Kolev, SD

Title:

Development of a portable 3D-printed flow-through passive sampling device free of flow pattern effects

Date:

2018-12-01

\section{Citation:}

Nitti, F., Almeida, M. I. G. S., Morrison, R., Cattrall, R. W., Pettigrove, V. J., Coleman, R. A. \& Kolev, S. D. (2018). Development of a portable 3D-printed flow-through passive sampling device free of flow pattern effects. MICROCHEMICAL JOURNAL, 143, pp.359-366. https:// doi.org/10.1016/j.microc.2018.08.029.

Persistent Link:

http://hdl.handle.net/11343/291518 